This is the accepted version of the article:

Stepanow, S.; Lodi Rizzini, A.; Krull, C.; Kavich, J.; Cezar, J.C.; Yakhou-Harris, F.; Sheverdyaeva, P.M.; Moras, P.; Carbone, C.; Ceballos, G.; Mugarza, A.; Gambardella, P.. Spin tuning of electron-doped metal-phthalocyanine layers. Journal of the American Chemical Society, (2014). 136. 14: 5451 - . 10.1021/ja501204q.

Available at: https://dx.doi.org/10.1021/ja501204q 


\title{
Spin tuning of electron-doped metal-phthalocyanine layers
}

\author{
Sebastian Stepanow, ${ }^{*, \perp}$ Alberto Lodi Rizzini,,${ }^{\prime \prime}$ Cornelius Krull," Jerald Kavich," Julio C. Cezar, ${ }^{\S, \ddagger}$ Flora \\ Yakhou-Harris, ${ }^{\S}$ Polina M. Sheverdyaeva, ${ }^{\#}$ Paolo Moras, ${ }^{\#}$ Carlo Carbone, ${ }^{\#}$ Gustavo Ceballos," Aitor \\ Mugarza," Pietro Gambardella ${ }^{\perp, \|}$
}

${ }^{\perp}$ Department of Materials, ETH Zürich, Hönggerbergring 64, 8093 Zürich, Switzerland.

"Catalan Institute of Nanoscience and Nanotechnology (ICN2), UAB Campus, 08193 Barcelona, Spain.

${ }^{\S}$ European Synchrotron Radiation Facility, BP 220, 38043 Grenoble, France.

\#Istituto di Struttura della Materia, Consiglio Nazionale delle Ricerche, 34012 Trieste, Italy.

\begin{abstract}
The spin state of organic-based magnets at interfaces is to a great extent determined by the organic evironment and the nature of the spin carrying metal center, which is further subject to modifications by the adsorbate-substrate coupling. Direct chemical doping offers an additional route for tailoring the electronic and magnetic characteristics of molecular magnets. Here we present a systematic investigation of the effects of alkali metal doping on the charge state and crystal field of $3 \mathrm{~d}$ metal ions in $\mathrm{Cu}, \mathrm{Ni}$, $\mathrm{Fe}$, and $\mathrm{Mn}$ phthalocyanine (Pc) monolayers adsorbed on Ag. Combined x-ray absorption spectroscopy and ligand field multiplet calculations show that $\mathrm{Cu}(\mathrm{II}), \mathrm{Ni}(\mathrm{II})$, and $\mathrm{Fe}(\mathrm{II})$ ions reduce to $\mathrm{Cu}(\mathrm{I}), \mathrm{Ni}(\mathrm{I})$, and $\mathrm{Fe}(\mathrm{I})$ upon alkali metal adsorption, whereas $\mathrm{Mn}$ maintains its formal oxidation state. The strength of the crystal field at the $\mathrm{Ni}, \mathrm{Fe}$, and $\mathrm{Mn}$ sites is strongly reduced upon doping. The combined effect of these changes is that the magnetic moment of high and low spin ions such as $\mathrm{Cu}$ and $\mathrm{Ni}$ can be entirely turned off or on, respectively, whereas the magnetic configuration of $\mathrm{MnPc}$ can be changed from intermediate (3/2) to high (5/2) spin. In the case of FePc a tenfold increase of the orbital magnetic moment accompanies charge transfer and a transition to a high spin state.
\end{abstract}

\section{INTRODUCTION}

Phthalocyanines $(\mathrm{Pc})$ are stable planar molecules that are interesting for their similarity to the biological relevant porphyrines and are increasing in technological importance because of their electrical, optical and catalytic properties. ${ }^{1-4}$ In general, Pc forms complexes by substitution of the two weakly acidic hydrogen atoms at inner nitrogen sites by divalent positively charged coordination centers. The fourfold chelating nitrogen ligand forms stable complexes with nearly all metals and halfmetals. In most cases the metal center resides within the plane of the macrocycle and is subject to a relatively strong crystal field imposed by the four inner pyrrole nitrogen atoms. As a consequence, the metal ions tend to be found in low or intermediate spin states sensitive to changes in the local chemical environment. ${ }^{5}$

Already in the early stages after the discovery of the phthalocyanines, researchers investigated modifications of the complexes by further oxidation and reduction of the metal center and Pc ligand. ${ }^{6-10}$ Remarkably, phthalocyanines allow not only the inclusion of many different metal ions but also in a much broader variety the stepwise reduction or oxidation of the complexes up to several times without disturbing the structure. This opens the possibility to control the total number of electrons in the complex.

The planar structure of the complex enables further ligation at axial positions. This fact was used to modify the magnetic response through the formation of adducts for surface-supported phthalocyanines and porphyrine derivatives with small molecular switches. ${ }^{11-14}$ The addition of molecules to the metal center leads to charge redistribution mainly due to modification of the ligand field but also possible by direct oxidation of the metal centers. ${ }^{15}$

Recently, electron-doped thin phthalocyanine films have gained interest for applications such as organic field effect transistors, ${ }^{16,17}$ light-emitting devices ${ }^{18}$ and photovoltaic cells, ${ }^{19}$ as well as for fundamental studies of correlated electron effects ${ }^{20,21}$. Although the electronic and magnetic properties of Pc anions have been discussed in the early stages of the discovery of stable multiply reduced metal-phthalocyanines, the precise nature of the charge and spin configuration was under debate for a long time..$^{7-9}$ Photoemission, electron energy-loss spectroscopy and near-edge x-ray absorption fine structure investigations clearly show shifts in the LUMO energy position associated with work function changes. ${ }^{22-29}$ The charging sequence of the metal-phthalocyanines (filling of orbitals for $\mathrm{CuPc}$ and $\mathrm{NiPc}$ is limited to ligand states only, wheras for FePc and $\mathrm{CoPc}$ metal ion centered states are also involved) upon doping of the thin films ${ }^{22,30}$ was found to agree with earlier reported assignments for bulk compounds ${ }^{7}$ and in electrochemical experiments ${ }^{9}$. Measurements of the electrical conductance of alkali-metal doped metal phthalocyanine films revealed an insulator-metalinsulator transition, which was attributed to the progressive filling of the four-fold degenerate LUMO in CuPc and NiPc, whereas $\mathrm{MnPc}, \mathrm{FePc}$, and $\mathrm{CoPc}$ showed a more complex behavior. ${ }^{16,17}$

Doping of phthalocyanine chemisorbed on a metal substrate is further complicated due to the interplay of additional substrate induced charging and screening effects that can alter the role of the charge donor towards the molecules. ${ }^{31-41}$ In a recent systematic investigation, the step-wise attachment of $\mathrm{Li}$ atoms to $\mathrm{CuPc}$ and $\mathrm{NiPc}$ molecules adsorbed on a $\mathrm{Ag}(001)$ surface was 
investigated by scanning probe microscopy and DFT calculations. ${ }^{42}$ The results demonstrate the existence of distinct doping sites where the effect of the dopant, i.e., charging of the ligand or metal center, depends crucially on its position on the molecule. Li dopants near the metal center $(\mathrm{Ni}$ or $\mathrm{Cu})$ donate electrons to the metal d-states, whereas Li dopants at benzyne or pyrrole sites donate electrons to the LUMO. Thus, depending on the doping site, different charge and spin configurations can be realized, which shows the flexibility of tuning the spin structure of organic-based magnets by non-magnetic dopants.
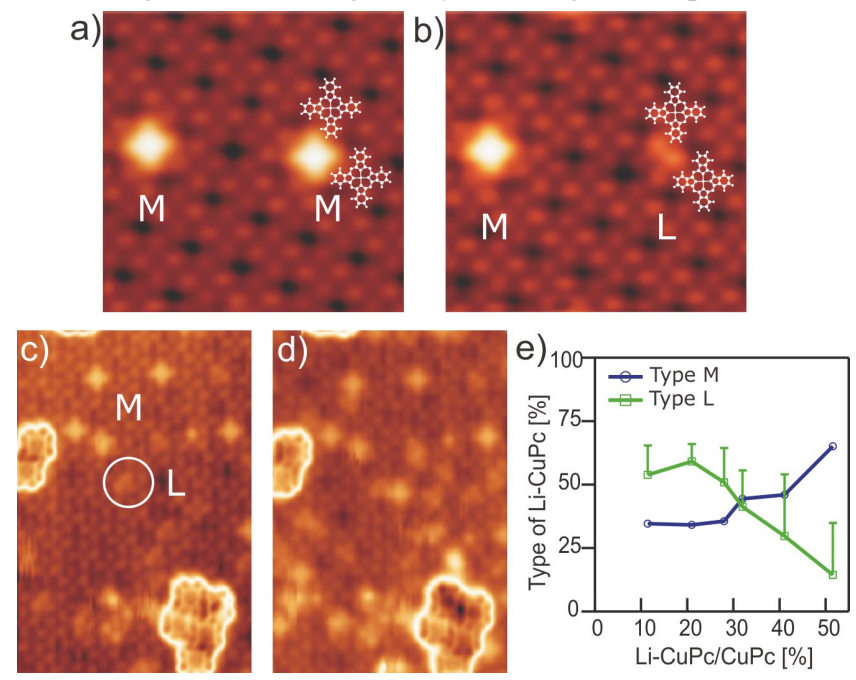

Figure 1. (a) STM topography of two doped CuPc complexes of $\mathrm{M}$ type within a $\mathrm{CuPc}$ monolayer $(0.17 \mathrm{nA},+0.2 \mathrm{~V}, 8.3 \mathrm{~nm} \times 8.3 \mathrm{~nm})$. (b) Topography of the same region after applying a voltage pulse ($2.2 \mathrm{~V}$ ) on the right $\mathrm{M}$ type, which converts into an L type. (c) Extended topography of a $\mathrm{CuPc}$ monolayer with low $\mathrm{Li}$ coverage. Molecules in the second adlayer are imaged in reverse contrast $(0.17 \mathrm{nA}, 0.2 \mathrm{~V}, 18.5 \mathrm{~nm} \times 12.7 \mathrm{~nm})$. (d) STM image of the same region with higher Li coverage. (e) Statistical analysis of the type of dopant as a function of Li coverage extracted from the STM data (total number of complexes $=500$ ). The error bars account for undefined configurations.

The effects of alkali metal doping on the magnetic properties of metal-phthalocyanines, however, have not been studied in a systematic way, neither in the bulk ${ }^{43,44}$ nor at interfaces. Such effects are important when considering the formation of electrical contacts between phthalocyanine molecules and a metallic electrode, ${ }^{42}$ and as well in understanding spin injection efficiency $^{45}$ and spin coupling ${ }^{46}$ between phthalocyanines and ferromagnetic metal layers.

In this study we focus on the effect of Li doping on the electronic and magnetic states of the metal ions for a series of $3 \mathrm{~d}$ metal-phthalocyanine monolayers ( $\mathrm{MnPc}, \mathrm{FePc}, \mathrm{NiPc}, \mathrm{CuPc}$ ) adsorbed on $\operatorname{Ag}(001)$. The systematic investigation presented here employs x-ray magnetic circular dichroism complemented by atomic multiplet calculations allowing us to accurately determine changes of the electronic valence and crystal field that result in a profound impact on the magnetic properties of the metal centers. The charging behavior is distinct from alkali metal doped thin films and bulk compounds. It shows readily reduction of the metal ions for $\mathrm{CuPc}$ and NiPc accompanied by the quench or emergence of a magnetic moment, respectively. FePc is also reduced by $\mathrm{Li}$ attachment, but is accompanied by a strong reduction of the crystal field potential. The same strong reduction of the ligand field occurred for $\mathrm{MnPc}$; however no changes of the metal ion valence state was observed. This results in an enhanced magnetic response due to a spin-state transition from intermediate to high-spin for both $\mathrm{FePc}$ and $\mathrm{MnPc}$. The results demonstrate that alkali metal doping is an efficient route to tune the spin properties of organic-based magnets at interfaces.

\section{METHODS}

STM. The STM measurements shown in Figure 1 were carried out at a base temperature of $4.8 \mathrm{~K}$, after depositing a CuPc monolayer on a single crystal $\operatorname{Ag}(100)$ substrate at $300 \mathrm{~K} . \mathrm{Li}$ atoms were deposited in-situ during scanning using a thermally heated SAES Getters source.

XAS. The experiments were carried out at the ID08 beamline of the European Synchrotron Radiation Facility (ESRF). The samples were prepared in-situ by sublimation of metal-phthalocyanines onto the clean $\operatorname{Ag}(001)$ surface held at $300 \mathrm{~K}$. The samples were first characterized by room-temperature STM and then transferred to the XMCD chamber entirely under UHV conditions. X-ray absorption spectra were acquired in total electron yield for circular and linear polarization in magnetic fields up to $5 \mathrm{~T}$ collinear with the $\mathrm{x}$-ray direction. The XAS spectra were measured for $\mathrm{X}$-ray incidence angles $\theta$ normal $\left(\theta=0^{\circ}\right)$ and grazing $\left(70^{\circ}\right)$ with respect to the surface normal. The XAS data acquired with vertical and horizonzal linear polarization corresponds to the E-vector aligned parallel to the surface and nearly perpendicular to the surface making an angle of $20^{\circ}$ with the surface normal, respectively. The sample temperature was varied between 300 and $8 \mathrm{~K}$. Li was deposited from a SAES Getters source onto the samples in the XMCD chamber at $8 \mathrm{~K}$ with the magnetic field turned off. The Li doping was carried out in several steps and after each deposition the XAS was recorded at the L-edges of the metal ions to follow the changes in their electronic structure. Due to the inherent averaging nature of the method we present here only data for which no further changes were observed upon $\mathrm{Li}$ doping. Hence it is reasonable to assume that all metal ions assume the same electronic configuration.

Multiplets. The atomic multiplet model takes into account the electron-electron interaction among $\mathrm{d}$ - and $\mathrm{p}$-electrons using rescaled Slater-Condon integrals and the atomic spin-orbit interaction. ${ }^{47-49}$ The molecular environment is simulated by a crystal field potential with $\mathrm{C}_{4 \mathrm{v}}$ symmetry. The Hamiltonian for the initial and final state is diagonalized considering all contributions (electron-electron interaction, crystal field, spin-orbit coupling and magnetic field) simultaneously. The intensity of the X-ray absorption spectra is calculated in the dipole approximation within Fermi's Golden rule. The finite temperature is taken into account by Boltzman weighted population of the first excited states of the initial state configuration. The obtained energies and amplitudes of the transitions are broadened using a Lorentzian and Gaussian function to simulate internal lifetime and external experimental energy broadening. To capture the most relevant chemical properties only a single d-shell configuration is considered for each set of data. To obtain good agreement between simulated and experimental XAS spectra the crystal field parameters for the final state were reduced by 10 $20 \%$ as detailed in the Supplemental Information (SI). The complete set of parameters for the multiplet calculations and the detailed results for each metal center can also be found in the SI. Orbital occupation and wavefunction symmetry analysis was performed in terms of the unperturbed gas phase $\mathrm{D}_{4 \mathrm{~h}}$ symmetry 
states to make the analysis comparable with previous studies (see Table 1).
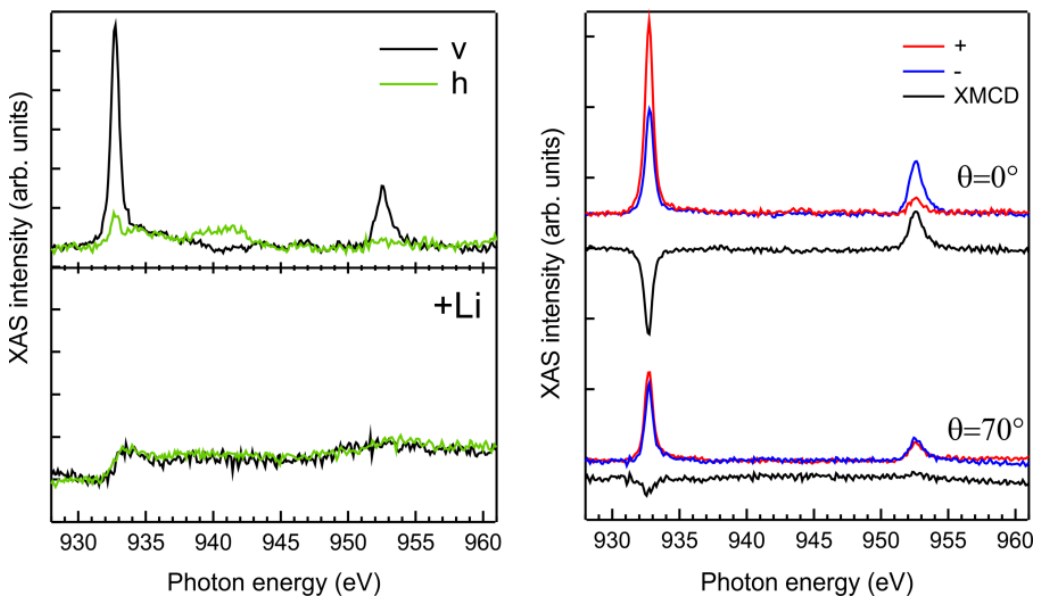

Figure 2. XAS spectra for $\mathrm{CuPc}$ monolayer on $\mathrm{Ag}(001)$. (a) Linear polarized XAS spectra for pristine (upper panel) and Li doped adlayer (bottom). (The E-vector is (v) parallel or (h) nearly perpendicular to the surface.) (b) Circular polarized XAS for the pristine layer and corresponding $\mathrm{XMCD}$ for normal $\left(0^{\circ}\right)$ and grazing $\left(70^{\circ}\right)$ x-ray incidence angle $(T=8 \mathrm{~K}, B=5 \mathrm{~T})$.

\section{RESULTS AND DISCUSSION}

Structure. Before discussing the XAS data we present results from a scanning tunneling microscopy investigation of the Li doping site dependence of a $\mathrm{CuPc}$ monolayer on $\operatorname{Ag}(001)$ (see Figure 1). The CuPc arrange in homochiral close-packed arrays on the Ag terraces. ${ }^{50}$ The addition of a small amount of $\mathrm{Li}$ atoms leads to two major types of $\mathrm{Li}-\mathrm{CuPc}$ complexes analogue to the structures found for single CuPc adsorbates. ${ }^{42}$ The $\mathrm{Li}$ atoms can reside at the phthalocyanine ligand position (denoted L) or close to the metal center (denoted M). The latter configuration appears symmetric and bright in the STM topographs as expected for Li occupying a central position. The two configurations are not equally stable and their relative frequency depends on the Li coverage. At low coverage both types can be found with more preference for the L-type. By applying a voltage pulse to the STM tip it is possible to irreversibly switch the type M to a type L, as can be seen in Figure 1 (a) and (b). This suggests that the type $M$ complex is less stable than type L. On the other hand, with increasing Li coverage we observe an increase in the $\mathrm{M}$ configuration that eventually becomes the dominant adsorption site even though there are more ligand sites available than central positions (Figure 1c). A possible explanation for this behavior is the electrostatic repulsion among Li ions, which is reduced when they are spaced apart and surface dipoles are more localized. The preference of the Li atoms for the metal sites in extended $\mathrm{CuPc}$ layers at high coverage shows that alkali metal doping may induce different effects for different concentrations of dopants. For this reason, all the XAS results presented in this work are taken in the saturation limit, i.e., at high Li coverage.

CuPc. Figure 2 shows the XAS and XMCD results for a $\mathrm{CuPc}$ monolayer. The data and results for the pristine $\mathrm{CuPc}$ monolayer were already discussed in detail in Ref. 51. The Ledge spectra of $\mathrm{Cu}$ show two single peaks, whose intensity depends strongly on the $\mathrm{x}$-ray incidence angle. This is due to a single hole in the $\mathrm{Cu}$ d-shell residing predominatly in the $\mathrm{d}_{\mathrm{x} 2-\mathrm{y} 2}$ $\left(b_{1 g}\right)$ orbital. Hence, the $\mathrm{Cu}$ ion has spin moment $1 / 2$ as evidenced by the XMCD signal. Note that the apparent weak $\mathrm{XMCD}$ signal at grazing incidence is a result of the strongly anisotropic charge distribution in the d-shell leading to a dominating spin dipolar term in the XMCD intensity.

Upon deposition of $\mathrm{Li}$ atoms on top of the $\mathrm{CuPc}$ monolayer the white line intensity at the $\mathrm{Cu}$ L-edge decreases and eventually vanishes. The step-like features in the linear polarized XAS stem from isotropic transitions from the localized $\mathrm{Cu} 2 \mathrm{p}$ into continuum states. The absence of resonant $2 \mathrm{p}-3 \mathrm{~d}$ transitions signifies a completely filled $d$-shell. Thus, the magnetic moment of the Li doped CuPc is completely quenched. This result is in agreement with previous DFT calculations, which demonstrate that $\mathrm{Li}$ atoms attached to the $\mathrm{Cu}$ center donate one electron to the $\mathrm{d}$-shell yielding a non-magnetic $\mathrm{d}^{10}$ configuration. ${ }^{42}$ In contrast, the reduction of the molecules in distinct $\mathrm{K}_{\mathrm{x}} \mathrm{CuPc}$ films was ascribed to electron attachment to the phthalocyanine orbitals only. ${ }^{26}$ This seeming discrepancy may be resolved by the fact that in the film phases the doped potassium atoms reside on particular ligand positions thus donating the electron to the molecular LUMO orbitals. Only when heaviliy doped with sodium atoms a reduction of the $\mathrm{Cu}$ (II) to $\mathrm{Cu}$ (I) ions was observed, ${ }^{25}$ which, however, in earlier reports were associated with the substitution of the $\mathrm{Cu}$ ions by $\mathrm{Na}^{8}$

NiPc. The opposite effect of the Li doping for the magnetic moment can be observed for NiPc. The pristine NiPc monolayer shows a similar strong dependence on the x-ray incidence angle for the linear polarized photons as $\mathrm{CuPc}$ (Figure 3). However, in comparison with $\mathrm{CuPc}$ the XAS lineshape shows additional satellite features that are characteristic for a $\mathrm{d}^{8}$ electronic configuration. The multiplet simulations presented in Fig. $3 \mathrm{c}$ and d show good agreement with the experimental data assuming 8 electrons in the d-shell. The calculations show that Ni has two holes in the $\mathrm{d}_{\mathrm{x} 2-\mathrm{y} 2}\left(\mathrm{~b}_{1 \mathrm{~g}}\right)$ orbital due to a strong crystal field splitting of the d-states, i.e. a large $10 \mathrm{D}_{\mathrm{q}}$ crystal field value $\left(10 \mathrm{D}_{\mathrm{q}}=2.3, \mathrm{D}_{\mathrm{s}}=0.8\right.$ and $\left.\mathrm{D}_{\mathrm{t}}=0.2 \mathrm{eV}\right)$. The strong level splitting also has a pronounced effect on the magnetic properties, i.e. $\mathrm{Ni}$ assumes a low-spin configuration with $S=0$. Accordingly, the experiments as well as the simulations show zero XMCD signal for the circular polarized XAS data. 
This situation changes when the NiPc adlayer is doped with

Li. With increasing concentration of Li atoms a new peak

LINEAR
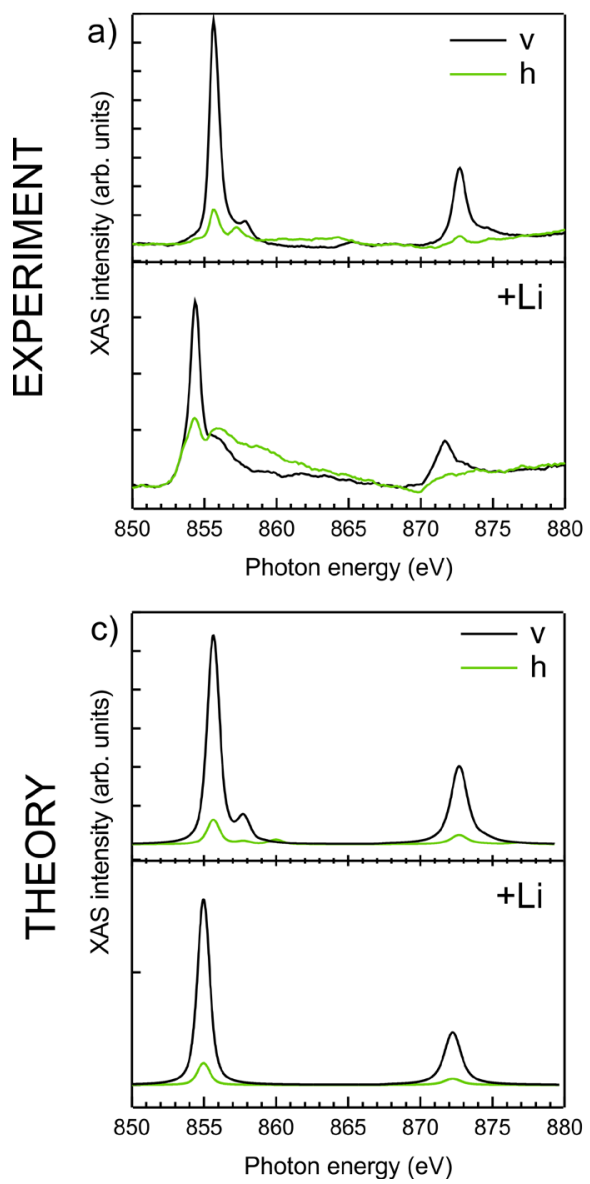

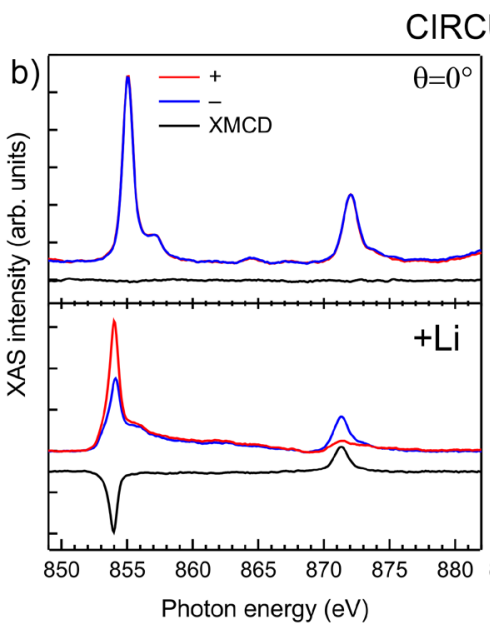

CIRCULAR
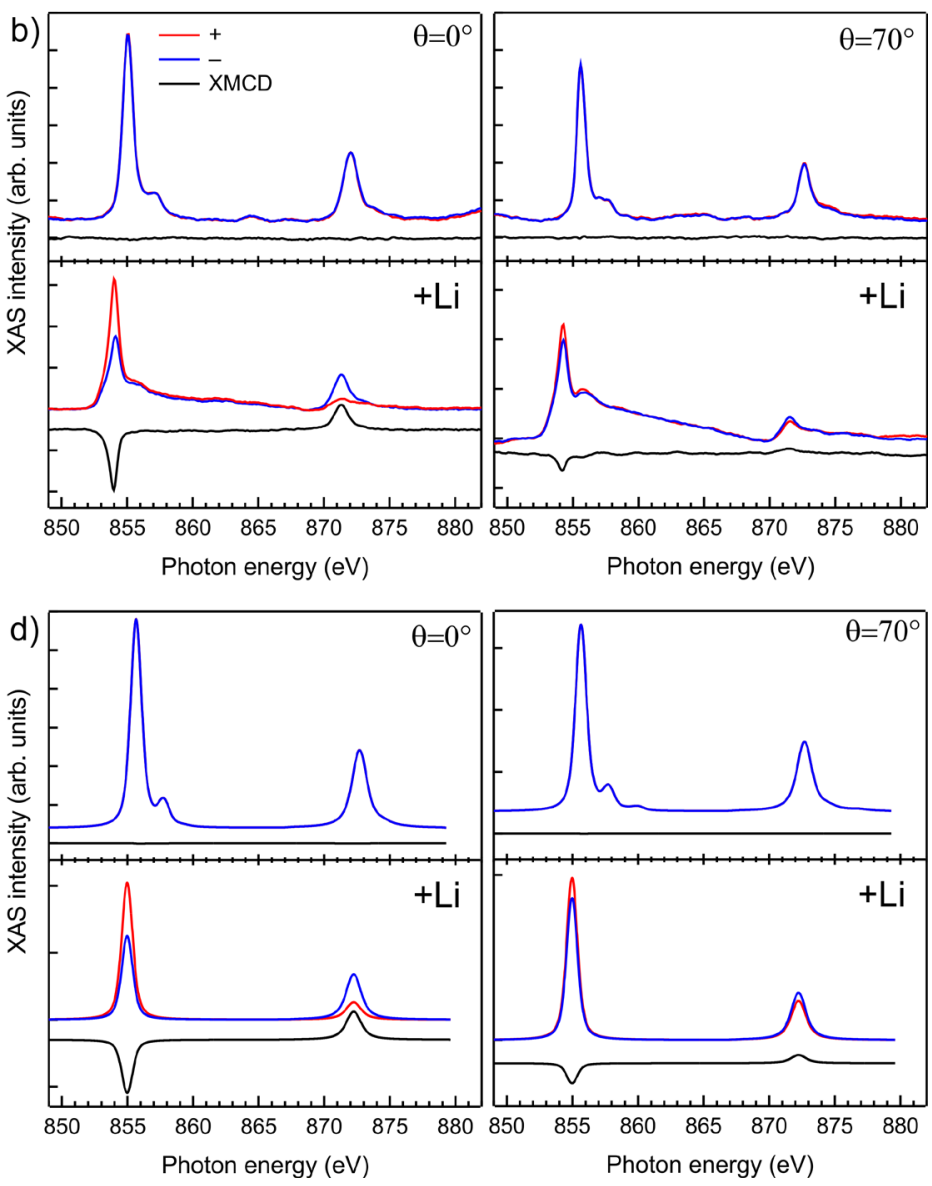

Figure 3. XAS spectra for NiPc monolayer on $\operatorname{Ag}(001)(T=8 \mathrm{~K}, B=5 \mathrm{~T})$. (a) XAS spectra for vertical (v) and horizontal (h) polarized light. (b) XAS data with circular polarization and corresponding XMCD intensity for $\left(0^{\circ}\right)$ normal and $\left(70^{\circ}\right)$ grazing incidence. The upper spectra correspond to the pristine and the lower spectra to the Li-doped NiPc adlayer. (c,d) Corresponding multiplet simulations of the experimental data in $(\mathrm{a}, \mathrm{b})$, respectively. The vertical ticks in all graphs correspond to the same spacing for comparison.

evolves at the low energy side of the former $\mathrm{L}_{3}$ main peak. The intensity of the new peak increases at the expense of the high energy peak (see Fig. S1 in supporting information (SI)). The linear polarized XAS data for the maximally doped NiPc layer is shown in the bottom row of Fig. $3 \mathrm{a}$ and b. The Li doped NiPc layer has a lower L-edge whiteline intensity indicating an increased d-shell occupancy. The shift to lower photon energies is also in accordance with a reduction of the Ni ions. In comparison with the $\mathrm{CuPc}$ the $\mathrm{NiPc}+\mathrm{Li}$ spectra show additional features, however at a position of the former peak of the pristine adlayer. The circular polarized XAS data for the doped layer demonstrates that the Ni ions have a finite magnetic moment. The XMCD signal is very similar to the CuPc layer both in its lineshape, relative strength to the XAS signal and its angular dependence. The circular polarized data also shows that the satellite intensity at the former pristine NiPc layer position has no XMCD signal. Thus we infer that the XAS satellite intensity is due to remaining undoped or L-type NiPc molecules and that the $\mathrm{Ni}$ ions have been reduced to a $\mathrm{d}^{9}$ electronic configuration.

The Li doped NiPc data was also simulated starting from a d ${ }^{9}$ configuration assuming that $\mathrm{Li}$ donates one electron to the $\mathrm{Ni}$ dstates. The crystal field parameters were adjusted to match the
$\mathrm{XMCD} \mathrm{L}_{3} / \mathrm{L}_{2}$ intensity ratio. The experimental data is well reproduced. One finds that the induction of a magnetic moment is due to the filling of one of the spin-paired holes in the $d_{x 2-y 2}$ orbitals yielding a free spin $1 / 2$.

Considering both $\mathrm{CuPc}$ and $\mathrm{NiPc}$ the Li doping has opposite effects. For $\mathrm{CuPc}$ the magnetic moment can be turned off while for NiPc the non-magnetic centers show a sizable magnetic moment after doping. This effect is ascribed to direct electron transfer from $\mathrm{Li}$ to the metal center at sufficiently high Li concentration levels which increases the d-level occupation by one.

FePc. A somewhat more intricate picture is found for FePc and $\mathrm{MnPc}$, where the holes are distributed over different metal dorbitals and thus electron correlation effects are expected to play a dominant role. The XAS and XMCD data for the pristine and Li-doped FePc monolayer is shown in Figure 4. The XAS data and multiplet analysis of a FePc monolayer interacting with a $\mathrm{Au}(111)$ surface is reported in Ref. 37. The XAS data for FePc adsorbed on $\operatorname{Ag}(001)$ differs somewhat in the number and position of the multiplet features from $\mathrm{FePc}$ on $\mathrm{Au}(111)$ signifying that the substrate-adsorbate chemical bonding and associated charge transfer plays a crucial role in the determination of the electronic structure. ${ }^{34,38,40,52}$ This is usually accompanied by a 

adsorbed on $\mathrm{Au}(111)$ the magnetic moment of the Fe center is nearly quenched on the $\operatorname{Ag}(001)$ surface as evidenced by the

LINEAR
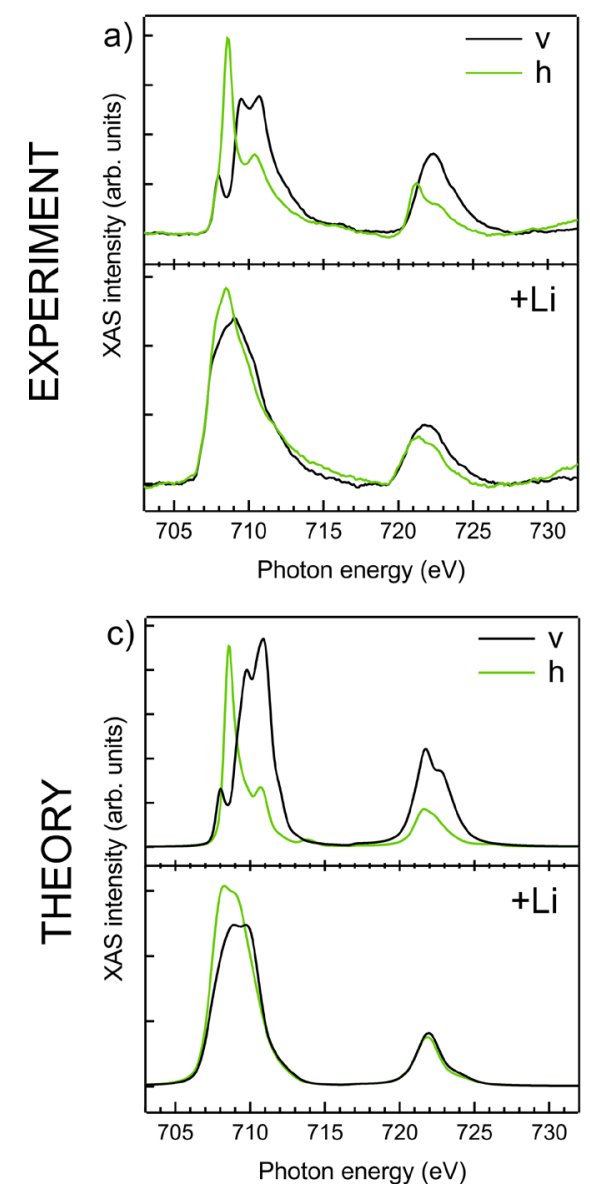
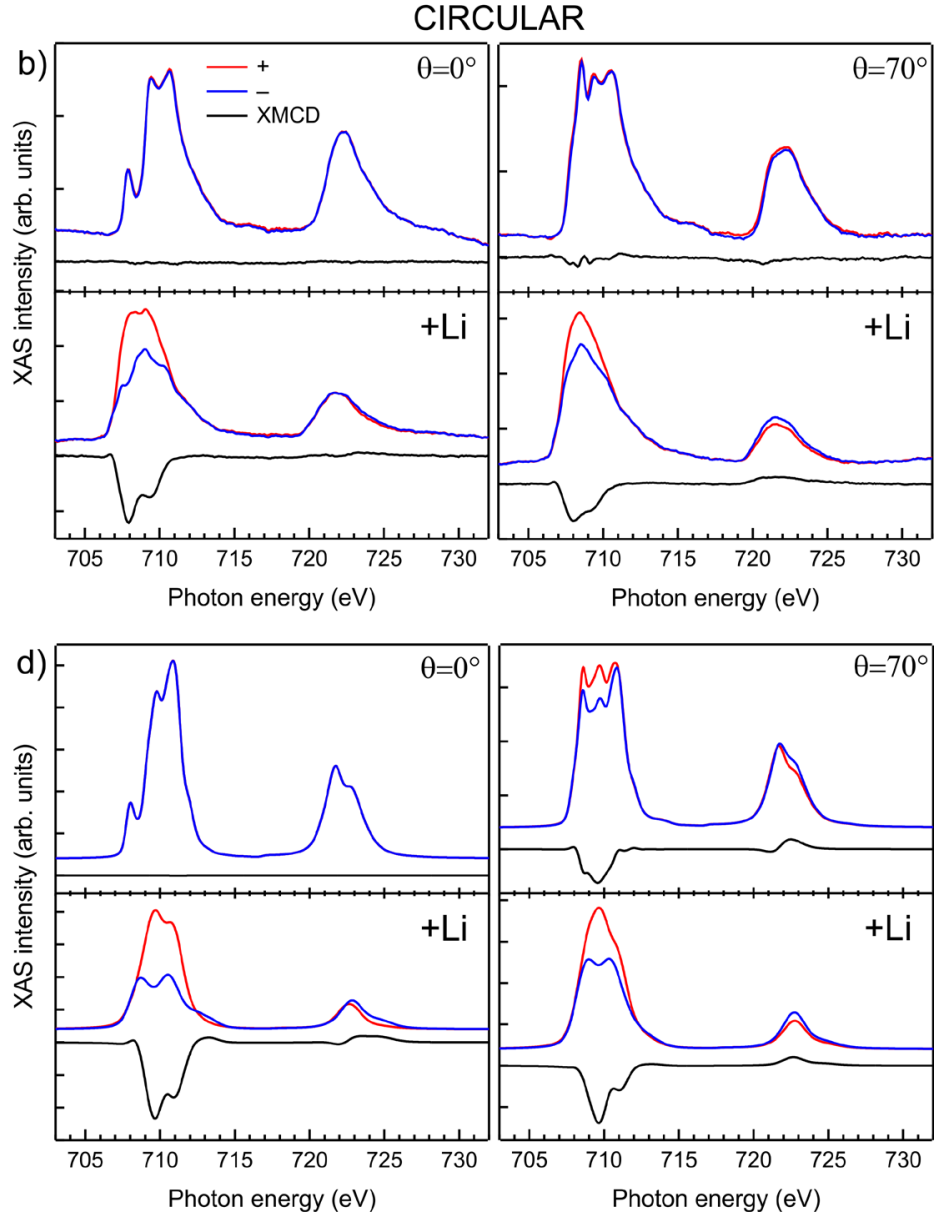

Figure 4. XAS spectra for FePc monolayer on $\operatorname{Ag}(001)(T=8 \mathrm{~K}, B=5 \mathrm{~T})$. (a) XAS spectra for vertical (v) and horizontal (h) polarized light. (b) XAS data with circular polarization and corresponding XMCD intensity for $\left(0^{\circ}\right)$ normal and $\left(70^{\circ}\right)$ grazing incidence. The upper spectra correspond to the pristine and the lower spectra to the Li-doped FePc adlayer. (c,d) Corresponding multiplet simulations of the experimental data in $(a, b)$, respectively. The vertical ticks in all graphs correspond to the same spacing for comparison.

intensity (Figure $4 \mathrm{~b}$ ). This could be due to an enhanced coupling of the Fe d-states with the Ag substrate electrons. ${ }^{37,38}$

The XAS lineshape can be well matched using a single $\mathrm{d}^{6}$ configuration as shown in Fig. $4 \mathrm{c}$ and d. Analyzing the ground state obtained from the multiplet calculations yields an intermediate spin $\mathrm{S}=1$ configuration with $\mathrm{B}_{2 \mathrm{~g}}$ symmetry and $\left(b_{2 g}\right)^{1}\left(e_{g}\right)^{4}\left(a_{1 g}\right)^{1}$ orbital occupation. The simulated XMCD at grazing incidence is overestimated, which signifies that, although the obtained spin multiplicity correctly describes the XAS lineshape, the observed magnitude of the spin moment is likely reduced by many-body screening or Kondo screening or screening effects due to the presence of the metal substrate. To account for these effects configurational mixing must be included in the multiplet calculations. ${ }^{37}$ In comparison on $\mathrm{Au}(111)$ a $\mathrm{S}=1$ with mixed $\mathrm{B}_{2 \mathrm{~g}}-\mathrm{E}_{\mathrm{g}}$ character was found where the $a_{1 g}$ orbital was nearly filled with the extra hole residing in the $e_{g}$ orbital corresponding closely to the ground state configuration of the unperturbed $\mathrm{FePc}$ molecule found in thin films. ${ }^{53-}$ ${ }^{56}$ The electronic configuration of FePc adsorbed on $\mathrm{Ag}(001)$ differs from the pristine molecule and hence doping of the adsorbate is expected to differ from crystalline or solvated FePc. ${ }^{7}$

The XAS and XMCD data for Li doped FePc is presented in the bottom row of Fig. $4 \mathrm{a}$ and $\mathrm{b}$. The XAS lineshape changes significantly and the linear polarized XAS data indicates a more isotropic spectral intensity distribution. More importantly, the relatively strong XMCD intensity demonstrates a sizable magnetic moment at the Fe centers. Similar to the NiPc, Li doping induces a strong magnetic moment in the $\mathrm{FePc} / \mathrm{Ag}(001)$ adsorbate system.

The details of the enhancement of the magnetic moment are revealed by the multiplet calculations. The simulated spectra shown in Fig. $4 \mathrm{c}$ and d (lower spectra) reproduce well the main features of the experimental data. The best agreement with the experimental data was obtained using a $\mathrm{d}^{7}$ electronic configuration, i.e., one electron transferred to the Fe d-shell. This additional electron does not simply fill up an empty orbital. Due to the strong intrashell electronic correlation the occupation of the remaining orbitals changes as well. We obtain the orbital occupation $\left(\mathrm{b}_{1 \mathrm{~g}}\right)^{1}\left(\mathrm{~b}_{2 \mathrm{~g}}\right)^{1.8}\left(\mathrm{e}_{\mathrm{g}}\right)^{3.1}\left(\mathrm{a}_{1 \mathrm{~g}}\right)^{1.1}$ with an overall $\mathrm{E}_{\mathrm{g}}$ symmetry. Li doping leads to the population of the in-plane $b_{1 \mathrm{~g}}$ and $b_{2 \mathrm{~g}}$ orbitals and creates a hole in the $\mathrm{e}_{\mathrm{g}}$ states opposite to the findings reported earlier for crystalline compounds. ${ }^{7}$ The $\mathrm{Li}$ attachment to the $\mathrm{FePc}$ adsorbate significantly reduces the crystal field strength imposed on the metal center $\left(10 \mathrm{D}_{\mathrm{q}}=1.0, \mathrm{D}_{\mathrm{s}}=0.048\right.$ and $\mathrm{D}_{\mathrm{t}}=0.038 \mathrm{eV}$ for $\mathrm{Li}$ doped FePc compared with $10 \mathrm{D}_{\mathrm{q}}=2.3$, $D_{s}=0.59$ and $D_{t}=0.144 \mathrm{eV}$ for the pristine macrocycle). Thus the 
recovery of the magnetic moment is in part due to a transition from an electronic intermediate to a high-spin configuration where the three holes in the d-shell create a total spin of $S=3 / 2$.
The orbital redistribution also creates a large orbital moment as evidenced by the strong $\mathrm{XMCD} \mathrm{L}_{3}$ intensity while the spin moment remains nearly unchanged. The ground state octuplet
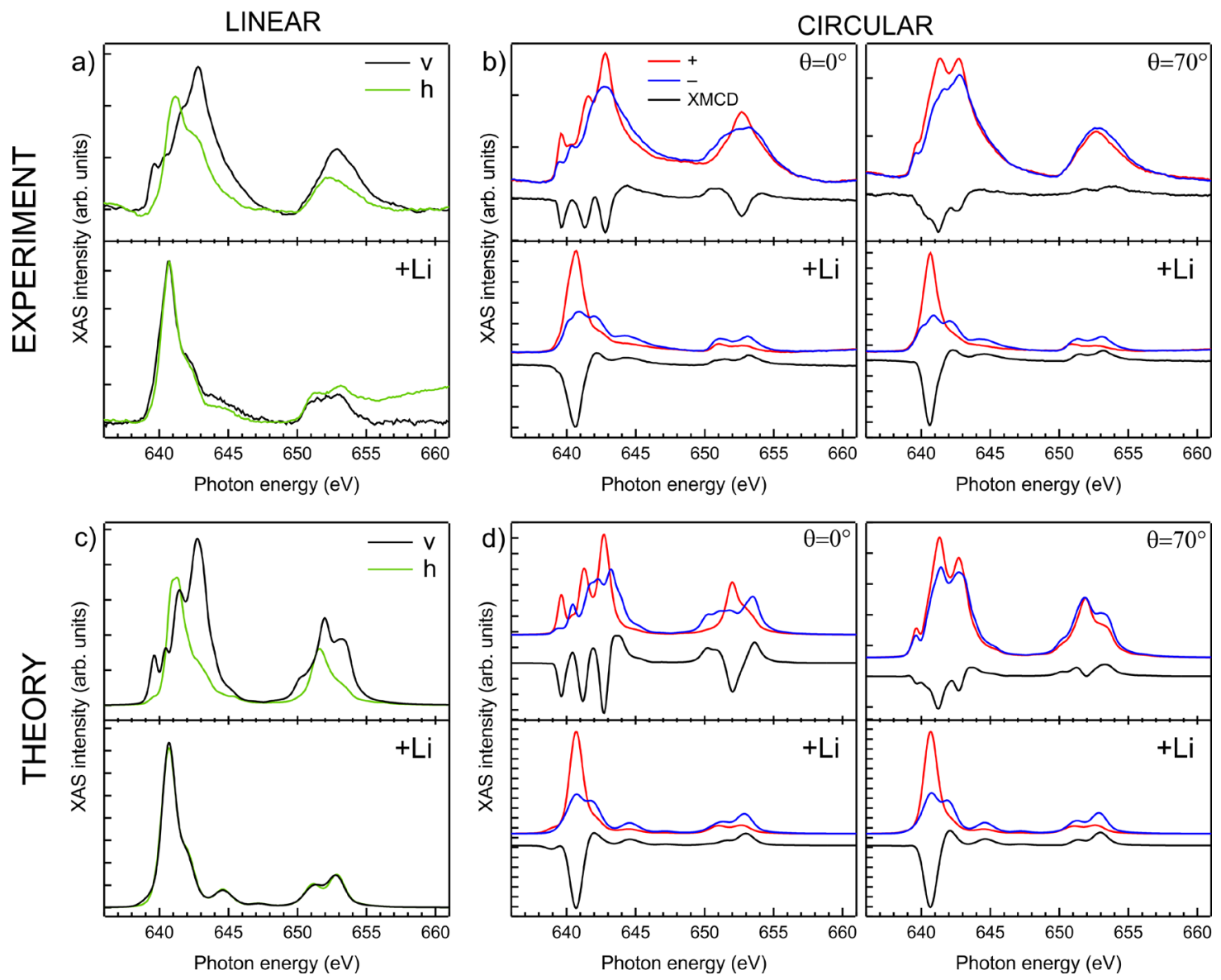

Figure 5. XAS spectra for MnPc monolayer on $\operatorname{Ag}(001)(T=8 \mathrm{~K}, B=5 \mathrm{~T})$. (a) XAS spectra for vertical (v) and horizontal (h) polarized light. (b) XAS data with circular polarization and corresponding XMCD intensity for $\left(0^{\circ}\right)$ normal and $\left(70^{\circ}\right)$ grazing incidence. The upper spectra correspond to the pristine and the lower spectra to the Li-doped MnPc adlayer. (c,d) Corresponding multiplet simulations of the experimental data in $(a, b)$, respectively. The vertical ticks in all graphs correspond to the same spacing for comparison.

manifold $(S=3 / 2 \otimes L=1)$ is strongly split and mixed with higher multiplet states by spin-orbit coupling, which reduces the ground state moments (see SI for more details). Although formally the d-shell occupation is that of a Co center, the orbital structure differs significantly due to the reduced crystal field potential, which is also reflected in the dissimilarity with the CoPc XAS spectra. ${ }^{37}$

Previously potassium intercalated FePc films grown on $\mathrm{Au}(001)$ were investigated by core level spectroscopy methods. It was concluded that $\mathrm{K}$ atoms donate electrons to both the molecular and $\mathrm{Fe} 3 \mathrm{~d}$ orbitals, ${ }^{28,57}$ in agreement with this study, although no precise result on the electronic configuration could be given.

MnPc. Contrary to the phthalocyanines discussed above, a different charging behavior is observed for MnPc adsorbed on $\operatorname{Ag}(001)$. The electronic structure of the Mn center in the intermediate $S=3 / 2$ configuration is very sensitive to its chemical environment, which is directly revealed by its rich XAS multiplet structure. ${ }^{35,58}$ The pristine $\mathrm{MnPc}$ monolayer on $\operatorname{Ag}(001)$ shows a strongly anisotropic lineshape for both XAS and
XMCD spectra presented in the top row of Fig. $5 \mathrm{a}$ and $\mathrm{b}$. The multiplet simulations (Fig. 5c,d) show good agreement with the experimental data. The relatively strong crystal field potential $\left(10 \mathrm{D}_{\mathrm{q}}=4.0, \mathrm{D}_{\mathrm{s}}=0.70\right.$ and $\left.\mathrm{D}_{\mathrm{t}}=0.52 \mathrm{eV}\right)$ results in an $\mathrm{S}=3 / 2$ intermediate spin configuration with orbital occupation $\left(b_{2 g}\right)^{1}\left(e_{g}\right)^{2}\left(a_{1 g}\right)^{2}$ having $B_{1 g}$ symmetry. The simulated out-ofplane XMCD intensity is significantly larger than the experimental signal and indicates potential charge transfer effects from the substrate. Upon Li doping, the XAS lineshape changes markedly. The spectra for linear polarization are nearly isotropic (Fig. 5a, bottom spectra) with much less multiplet features and narrower whiteline intensity distribution. The XMCD spectra of the Li doped MnPc reveals an enhanced magnetic moment in comparison with the pristine adlayer (Fig. 5b). The XAS and XMCD spectra with circular polarization are reminiscent of Mn high-spin ions and indeed best agreement with the multiplet calculations was found using a $\mathrm{d}^{5}$ configuration and a notably lowered crystal field potential $\left(10 \mathrm{D}_{\mathrm{q}}=1.0, \mathrm{D}_{\mathrm{s}}=-0.02\right.$, $\mathrm{D}_{\mathrm{t}}=0.09 \mathrm{eV}$ ) with nearly pure octahedral symmetry (see Fig. $5 \mathrm{c}, \mathrm{d})$. The reduction of the crystal field strength results in a high-spin configuration with orbital occupancies of 
$\left(b_{1 g}\right)^{1}\left(b_{2 g}\right)^{1}\left(e_{g}\right)^{2}\left(a_{1 g}\right)^{1}$. Thus the enhancement of the spin magnetic moment is entirely due to modifications of the chemical environment of the Mn centers without the reduction of the metal ion itself.

Alkali metal intercalated and doped MnPc films and bulk compounds have been previously investigated. ${ }^{27,44}$ For K-doped thin films it was demonstrated by electron energy-loss spectroscopy that for the $\mathrm{K}_{1} \mathrm{MnPc}$ phase potassium donates one electron to the $\mathrm{Mn} 3 \mathrm{~d}$ shell changing its valence and associated magnetic moment. ${ }^{27}$ Contrary to these results, for the major MnPc $\beta$-form in the bulk Iwasa et al. found no reduction of the Mn center when doped with Li up to 4 atoms per molecule. ${ }^{44}$ However, they observed a spin-state transition of the $\mathrm{Mn}^{2+}$ ions from intermediate spin to high-spin $S=5 / 2$ as we observed for the surface supported MnPc. The results can be reconciled considering that the structure of thin films differs from the bulk $\beta$-polymorph. This can result in different geometrical voids and interstitial sites that can create, in addition to the different ionic radii and ionization potentials of the $\mathrm{K}$ and $\mathrm{Li}$ ions, distinct dopant positions in these systems and hence a different doping behavior.

Considerations on crystal field and charge transfer. The marked lowering of the crystal field strength by Li doping and the apparent increase of the Slater-Condon parameters (see SI) is a surprising result as one would expect that the strong chemical bonding of the metal ions to the phthalocyanine base is very robust. The origin of this effect remains speculative. It is not clear if a single $\mathrm{Li}$ atom attached to the metal site is able to cause this effect or if the concerted action of several alkali dopants donating electrons to the ligand and metal centers simultaneously can account for this observation.

The experimental data represent a saturated final state upon which further doping does not change the state of the central metal atoms. The presence of a few positively charged Li ions nearby the metal centers may reduce their crystal field potential imposed by the negative pyrrole nitrogen atoms, while the donated electron charges are delocalized and well-screened within the phthalocyanine molecular orbitals and substrate states. Another scenario may involve atomic relaxation of the metal center's position out-of-plane of the phthalocyanine complex as the $\mathrm{Li}$ ions may compete with the bonding to the inner pyrrole nitrogen atoms. In this latter case the $\mathrm{Li}$ doping goes beyond its expected role of electron donation as it additionally induces structural changes due to its participation in the chemical bonding. The geometric distortions likely explain not only the reduction of the crystal field strength but also the appearance of nearly isotropic electronic configurations. Alkali metal reduced metal-phthalocyanines have been studied some time ago and it is known that at sufficiently high concentrations of, e.g., sodium atoms $\mathrm{Cu}$ metal centers can be replaced by the alkali ions. ${ }^{8}$ However, structural changes of the macrocycle were not observed in the crystalline alkali metal intercalated compounds discussed above.

The results emphasize the distinct site specific doping behavior of alkali metal atoms for metal-phthalocyanines which likely explains the differences observed between the alkali-metal intercalated thin film and bulk phases compared to the adlayers presented here. The former compounds may prefer ligand intercalation positions due to well-defined interstitial sites and bulk chemical environment.

Finally, although XAS is only directly sensitive to the electronic state of the metal centers, we wish to discuss the observed electron attachment to the metal-phthalocyanines in view of the macrocycle's orbital structure provided by recent systematic DFT calculations. ${ }^{38,59,60}$ The reduction of the phthalocyanines can involve metal ion or ligand centered states. The ligand $2 \mathrm{e}_{\mathrm{g}}$ orbitals are relatively strongly delocalized and have less Coulomb repulsion than the central atom $\mathrm{a}_{1 \mathrm{~g}}$ and $\mathrm{b}_{2 \mathrm{~g}}$. Charging of the central metal atoms is possible due to the reduction of Coulomb repulsion in the complex by delocalization of the electronic charge over the whole complex due to the $\pi$-interaction mediated mixing of central atom and ligand states. Hence, strictly speaking, one can only talk of the reduction of the whole complex, although the changes in the electronic structure of the MPc adsorbates can be discussed within a formal oxidation state picture. Note, that XAS is only directly sensitive to the electronic state of the metal centers including their electron occupation as well as the local crystal field potential imposed by the Pe ligand.

For $\mathrm{CuPc}$ and NiPc DFT calculations yield low lying unoccupied orbitals with ligand centered $2 \mathrm{e}_{\mathrm{g}}$ and with predominant metal $d_{x 2-y 2}\left(b_{1 g}\right)$ symmetry. Theory predicts in gas-phase the occupation of ligand states in the initial reduction steps. However, it can be expected that at certain doping concentrations electrons are transferred to the central metal ions due to the enhanced Coulomb screening provided by the substrate electrons. Moreover, the substrate acts as an electron donor to the phthalocyanine base already in the adsorption process. ${ }^{38,42}$ Furthermore, the attachment of $\mathrm{Li}$ atoms to specific sites on the macrocycles shows preference for electron transfer to either metal or ligand centered orbitals ${ }^{42}$ and hence the reduction sequence is not necessarily following the predictions for gas-phase anions. ${ }^{59}$ Thus the XAS data confirms the previous DFT and STM results. $^{42}$

Charging of the MnPc and FePc molecules is more involved. In an electrochemical environment, filling of metal $\mathrm{e}_{\mathrm{g}}$ and $\mathrm{a}_{1 \mathrm{~g}}$ orbitals occurs at some reduction step for $\mathrm{MnPc}$ and $\mathrm{FePc}$, 7,9 which is supported by the DFT analysis. ${ }^{59,60}$ However, the predicted DFT ground state occupations differ from the ones determined by the multiplet calculations. This may be a result of the adsorbate substrate coupling. ${ }^{38}$ The theoretical prediction of the $\mathrm{FePc}^{\mathrm{n}-}$ anionic molecules does not show an increase in the $\mathrm{Fe}$ magnetic moment but a decrease due to spin pairing upon charging. However, the calculations do not take into account the presence of counter ions or the metal substrate. The same scenario is observed for $\mathrm{MnPc}$ that according to theory should present a reduced Mn magnetic moment in its anionic state. Thus the observed reduction of the metal states for $\mathrm{CuPc}$ and NiPc as well as the observed spin-state transition to high-spin configurations for $\mathrm{MnPc}$ and $\mathrm{FePc}$ must be due to the specific electronic characteristics of the phthalocyanine adsorbate systems and offers a route to tune the magnetic properties in surface-supported organic-based magnets.

\section{CONCLUSIONS}

To summarize, the electronic structure and magnetic properties of a series ( $\mathrm{MnPc}, \mathrm{FePc}, \mathrm{NiPc}, \mathrm{CuPc}$ ) of metal-phthalocyanines adsorbed on $\operatorname{Ag}(001)$ and their doping with Li atoms was investigated by x-ray absorption spectroscopy and x-ray magnetic circular dichroism. The chemisorbed FePc and $\mathrm{MnPc}$ show some deviations from their pristine electronic structure upon adsorption, while $\mathrm{CuPc}$ and NiPc retain essentially their gas-phase electronic structure and magnetic properties. The deviations are ascribed to the finite adsorbate-substrate coupling. 
In general, Li doping leads to the onefold reduction of the metal centers with the exception of MnPc. This results in the quenching of the magnetic moment for $\mathrm{CuPc}$ and the recovery of a $\mathrm{S}=1 / 2$ state for NiPc. For MnPc and FePc a considerable reduction of the crystal field strength results in the transition from intermediate to high-spin systems. Alkali metal doping of the surface-supported metal-phthalocyanines is different from bulk intercalated compounds and must take into account the interplay of molecular hybridization and enhanced substrate Coulomb screening in the role of the electron donation from the alkali atoms to the macrocycle. The results demonstrate that the spin state of interfacial metal-phthalocyanines can be efficiently tuned by doping with $\mathrm{Li}$ atoms. The magnetic response can be either turned off or on in metal-Pc adsorbate systems with high or low spin configurations and can be enhanced for macrocycles with intermediate spin ground states.

Table 1. Ground state properties obtained by the multiplet calculations.

\begin{tabular}{|c|c|c|c|c|c|}
\hline Type & $d^{n}$ & $G S$ & $\begin{array}{l}\text { Electronic } \\
\text { configuration }\end{array}$ & $\begin{array}{l}<S_{z} \\
>\end{array}$ & $\begin{array}{l}<L_{z} \\
>\end{array}$ \\
\hline $\mathrm{MnPc}$ & 5 & $\begin{array}{l}\mathrm{B}_{1} \\
\mathrm{~g}\end{array}$ & $\left(b_{1 g}\right)^{0}\left(b_{2 g}\right)^{1}\left(e_{g}\right)^{2}\left(a_{1 g}\right)^{2}$ & $3 / 2$ & -0.05 \\
\hline $\begin{array}{l}\mathrm{MnPc}+\mathrm{L} \\
\mathrm{i}\end{array}$ & 5 & $\begin{array}{l}\mathrm{A}_{1} \\
\mathrm{~g}\end{array}$ & $\left(b_{1 g}\right)^{1}\left(b_{2 g}\right)^{1}\left(e_{g}\right)^{2}\left(a_{1 g}\right)^{1}$ & $5 / 2$ & 0 \\
\hline $\mathrm{FePc}$ & 6 & $\begin{array}{l}\mathrm{B}_{2} \\
\mathrm{~g}\end{array}$ & $\left(b_{1 g}\right)^{0}\left(b_{2 g}\right)^{1}\left(e_{g}\right)^{4}\left(a_{1 g}\right)^{1}$ & $1^{\mathrm{a}}$ & 0.08 \\
\hline $\mathrm{FePc}+\mathrm{Li}$ & 7 & $\mathrm{Eg}_{\mathrm{g}}$ & $\left(b_{1 g}\right)^{1}\left(b_{2 g}\right)^{1.8}\left(e_{g}\right)^{3.1}\left(a_{1 g}\right)^{1}$ & $1.1^{\mathrm{b}}$ & 0.8 \\
\hline $\mathrm{NiPc}$ & 8 & $\begin{array}{l}\mathrm{A}_{1} \\
\mathrm{~g}\end{array}$ & $\left(b_{1 g}\right)^{0}\left(b_{2 g}\right)^{2}\left(e_{g}\right)^{4}\left(a_{1 g}\right)^{2}$ & 0 & 0 \\
\hline $\mathrm{NiPc}+\mathrm{Li}$ & 9 & $\begin{array}{l}\mathrm{B}_{1} \\
\mathrm{~g}\end{array}$ & $\left(b_{1 g}\right)^{1}\left(b_{2 g}\right)^{2}\left(e_{g}\right)^{4}\left(a_{1 g}\right)^{2}$ & $1 / 2$ & 0.28 \\
\hline $\mathrm{CuPc}$ & 9 & $\begin{array}{l}\mathrm{B}_{1} \\
\mathrm{~g}\end{array}$ & $\left(b_{1 g}\right)^{1}\left(b_{2 g}\right)^{2}\left(e_{g}\right)^{4}\left(a_{1 g}\right)^{2}$ & $1 / 2$ & 0.2 \\
\hline $\mathrm{CuPc}+\mathrm{Li}$ & $\begin{array}{l}1 \\
0\end{array}$ & $\begin{array}{l}\mathrm{A}_{1} \\
\mathrm{~g}\end{array}$ & $\left(b_{1 g}\right)^{2}\left(b_{2 g}\right)^{2}\left(e_{g}\right)^{4}\left(a_{1 g}\right)^{2}$ & 0 & 0 \\
\hline
\end{tabular}

${ }^{\mathrm{a}}$ Note that the experimental magnetic moment is nearly zero.

${ }^{b}$ The lowering from $3 / 2$ is due to mixing with higher multiplets.

\section{ASSOCIATED CONTENT}

Supporting Information. NiPc XAS spectral evolution for subsequent Li atom deposition. Full list of employed multiplet parameters. Results (orbital occupation, magnetic moments \& symmetry of states) of the multiplet calculations for the lowest spin-orbit multiplet. This material is available free of charge via the Internet at http://pubs.acs.org.

\section{AUTHOR INFORMATION}

\section{Corresponding Author}

*sebastian.stepanow@mat.ethz.ch.

\section{Present Addresses}

†Dipartimento di Scienze Fisiche, Informatiche e Matematiche, Università di Modena e Reggio Emilia, Modena, Italy. ‡LNLS (Brazilian Synchrotron Radiation Laboratory), CNPEM, CP6192, 13083-970, Campinas, SP, Brazil.

\section{Author Contributions}

The manuscript was written through contributions of all authors. All authors have given approval to the final version of the manuscript.

\section{Funding Sources}

We acknowledge support from Ministerio de Ciencia e Innovación (MAT2010-15659), Agència de Gestió d'Ajuts Universitaris i de Recerca (2009 SGR 695), J. J. K. acknowledges funding through the National Science Foundation International Research Fellowship.

\section{ACKNOWLEDGMENT}

We thank R. Robles for fruitful discussions about the DFT results for Li-doped metal-phthalocyanines.

\section{ABBREVIATIONS}

Pc phthalocyanine; XAS x-ray absorption spectroscopy; XMCD xray magnetic circular dichroism; STM scanning tunneling microscopy.

\section{REFERENCES}

(1) McKeon, N. B. Phthalocyanine Materials; Cambridge University Press: Cambridge, 1998.

(2) Inabe, T.; Tajima, H. Chem. Rev. 2004, 104, 5503.

(3) Mack, J.; Kobayashi, N. Chem. Rev. 2011, 111, 281.

(4) Sorokin, A. B. Chem. Rev. 2013, 113, 8152.

(5) Bartolomé, J. (eds.) Molecular Magnets; Springer-Verlag; Berlin Heidelberg, 2014; pp221-245.

(6) Taube, R.; Zach, M.; Stauske, K. A.; Heidrich, S. Zeitschrift für Chemie 1963, 3, 392.

(7) Taube, R. Zeitschrift für Chemie 1966, 6, 8 .

(8) Clack, D. W.; Hush, N. S.; Yandle, J. R. Chem. Phys. Lett. 1967, $1,157$.

(9) Clack, D. W.; Yandle, J. R. Inorg. Chem. 1972, 11, 1738.

(10) Clack, D. W.; Hush, N. S.; Woolsey, I. S. Inorg. Chim. Acta 1976, 19, 129.

(11) Wäckerlin, C.; Chylarecka, D.; Kleibert, A.; Müller, K.; Iacovita, C.; Nolting, F.; Jung, T. A.; Ballav, N. Nat. Commun. 2010 , 1,61 .

(12) Miguel, J.; Hermanns, C. F.; Bernien, M.; Krüger, A.; Kuch, W. J. Phys. Chem. Lett. 2011, 2, 1455.

(13) Isvoranu, C.; Wang, B.; Ataman, E.; Schulte, K.; Knudsen, J.; Andersen, J. N.; Bocquet, M.-L.; Schnadt, J. J. Phys. Chem. C 2011, 115,20201

(14) Strózecka, A.; Soriano, M.; Pascual, J. I.; Palacios, J. J. Phys. Rev. Lett. 2012, 109, 147202.

(15) Sumimoto, M.; Kaeashima, Y.; Fujimoto, H. Dalton Trans. 2009, 2009, 5737.

(16) Craciun, M. F.; Rogge, S.; Morpurgo, A. F. J. Am. Chem. Soc. 2005, 127, 122110.

(17) Craciun, M. F.; Rogge, S.; den Boer, M.-J. L.; Margadonna, S.; Prassides, K.; Iwasa, Y.; Morpurgo, A. F. Adv. Mater. 2006, 18, 320.

(18) Hung, L. S.; Tang, C. W. Appl. Phys. Lett. 1999, 74, 3209.

(19) Singh, V. P.; Singh, R. S.; Parthasarathy, B.; Aguilera; Anthony, J.; Payne, M. Appl. Phys. Lett. 2005, 86, 082106.

(20) Tosatti, E.; Fabrizio, M.; Tóbik, J.; Santoro, G. Phys. Rev. Lett. 2004, 93, 117002.

(21) Filibian, M.; Carretta, P.; Mozzati, M. C.; Ghigna, P.; Zoppellaro, G.; Ruben, M. Phys. Rev. Lett. 2008, 100, 117601.

(22) Gargiani, P.; Calabrese, A.; Mariani, C.; Betti, M. G. J. Phys. Chem. C 2010, 114, 12258.

(23) Calabrese, A.; Floreano, L.; Verdini, A.; Mariani, C.; Betti, M. G. Phys. Rev. B 2009, 79, 115446.

(24) Schwieger, T.; Peisert, H.; Golden, M. S.; Knupfer, M.; Fink, J. Phys. Rev. B 2002, 66, 155207.

(25) Ding, H.; Park, K.; Green, K.; Gao, Y. Chem. Phys. Lett. 2008, $454,229$. 
(26) Flatz, K.; Grobosch, M.; Knupfer, M. J. Chem. Phys. 2007, 126, 214702 .

(27) Mahns, B.; Roth, F.; Grobosch, M.; Zahn, D. R. T.; Knupfer, M. J. Chem. Phys. 2011, 134, 194504.

(28) Aristov, V. Y.; Molodtsova, O. V.; Maslyuk, V. V.; Vyalikh, D. V.; Bredow, T.; Mertig, I.; Preobrajenski, A. B.; Knupfer, M. Organic Electronics 2010, 11, 1461.

(29) Ding, H.; Gao, H. Organic Electronics 2010, 11, 1786.

(30) Aristov, V. Y.; Molodtsova, O. V.; Knupfer, M. Organic Electronics 2011, 12, 372.

(31) Peisert, H.; Knupfer, M.; Schwieger, T.; Auerhammer, J. M.; Golden, M. S.; Fink, J. J. Appl. Phys. 2002, 91, 4872.

(32) Peisert, H.; Knupfer, M.; Fink, J. Appl. Phys. Lett. 2002, 81, 2400.

(33) Grobosch, M.; Aristov, V. Y.; Molodtsova, O. V.; Schmidt, C.; Doyle, B. P.; Nannarone, S.; Knupfer, M. J. Phys. Chem. C 2009, 113 , 13219.

(34) Petraki, F.; Peisert, H.; Aygül, U.; Latteyer, F.; Uihlein, J.; Vollmer, A.; Chassé, T. J. Phys. Chem. C 2012, 116, 11110.

(35) Petraki, F.; Peisert, H.; Hoffmann, P.; Uihlein, J.; Knupfer, M.; Chassé, T. J. Phys. Chem. C 2012, 116, 5121.

(36) Petraki, F.; Peisert, H.; Biswas, I.; Aygül, U.; Latteyer, F.; Vollmer, A.; Chassé, T. J. Phys. Chem. Lett. 2010, 1, 3380

(37) Stepanow, S.; Miedema, P. S.; Mugarza A.; Ceballos, G.; Moras, P.; Cezar, J. C.; Carbone, C.; de Groot, F. M. F.; Gambardella P. Phys. Rev. B 2011, 83, 220401.

(38) Mugarza, A.; Robles, R.; Krull, C.; Korytár, R.; Lorente, N.; Gambardella, P. Phys. Rev. B 2012, 85, 155437.

(39) Mugarza, A.; Krull, C.; Robles, R.; Stepanow, S.; Ceballos, G.; Gambardella, P. Nat. Commun. 2011, 2, 490.

(40) Gargiani, P.; Angelucci, M.; Mariani, C.; Betti, M. G. Phys. Rev. B 2010, 81, 085412.

(41) Betti, M. G.; Gargiani, P.; Frisenda, R.; Biagi, R.; Cossaro, A.; Verdini, A.; Floreano, L.; Mariani, C. J. Phys. Chem. C 2010, 114, 21638.

(42) Krull, C.; Robles, R.; Mugarza, A.; Gambardella, P. Nat. Mater. 2013, 12, 337.

(43) Sharoyan, E. G.; Manukyan, A. S. J. Porphyrins Phthalocyanines 2005, 9, 846 .

(44) Taguchi, Y.; Miyake, T.; Margadonna, S.; Kato, K.; Prassides, K.; Iwasa, Y. J. Am. Chem. Soc. 2006, 128, 3313.
(45) Cinchetti, M.; Neuschwander, S.; Fischer, A.; Ruffing, A.; Mathias, S.; Wüstenberg, J.-P.; Aeschlimann, M. Phys. Rev. Lett. 2010, 104, 217602.

(46) Lodi Rizzini, A.; Krull, C.; Balashov, T.; Kavich, J. J.; Mugarza, A.; Miedema, P. S.; Thakur, P. K.; Sessi, V.; Klyatskaya, S.; Ruben, M.; Stepanow, S.; Gambardella, P. Phys. Rev. Lett. 2011, 107, 177205 .

(47) van der Laan, G.; Thole, B. T. Phys. Rev. B 1991, 43, 13401.

(48) van der Laan, G.; Kirkman, I. W. J. Phys.: Condens. Matter 1992, 4, 4189.

(49) de Groot, F. Coord. Chem. Rev. 2005, 249, 31.

(50) Mugarza, A.; Lorente, N.; Ordejón, P.; Krull, C.; Stepanow, S.; Bocquet, M.-L.; Fraxedas, J.; Ceballos, G.; Gambardella, P. Phys. Rev. Lett. 2010, 105, 115702.

(51) Stepanow, S.; Mugarza, A.; Ceballos, G.; Moras, P.; Cezar, J. C.; Carbone, C.; Gambardella, P. Phys. Rev. B 2010, 82, 014405.

(52) Hu, Z.; Li, B.; Zhao, A.; Yang, J.; Hou, J. G. J. Phys. Chem. C 2008, 112, 13650 .

(53) Thole, B. T.; van der Laan, G.; Butler, P. H. Chem. Phys. Lett. 1988, 149, 295

(54) Cook, P. L.; Liu, X.; Yang, W.; Himpsel, F. J. J. Chem. Phys. 2009, 131, 194701

(55) Bartolomé, J.; Bartolomé, F.; García, L. M.; Filoti, G.; Gredig, T.; Colesniuc, C. N.; Schuller, I. K.; Cezar, J. C. Phys. Rev. B 2010, 81, 195405.

(56) Kroll, T.; Kraus, R.; Schönfelder, R.; Aristov, V. Y.; Molodtsova, O. V.; Hoffmann, P.; Knupfer, M. J. Chem. Phys. 2012, 137,054306

(57) König, A.; Roth, F.; Kraus, R.; Knupfer, M. J. Chem. Phys. 2009, 130, 214503

(58) Kataoka, T.; Sakamoto, Y.; Yamazaki, Y.; Singh, V. R.; Fujimori, A.; Takeda, Y.; Ohkochi, T.; Fujimori, S.-I.; Okane, T.; Saitoh, Y.; Yamagami, H.; Tanaka, A. Solid State Commun. 2012, 152, 806.

(59) Liao, M.-S.; Scheiner, S. J. Chem. Phys. 2001, 114, 9780. 1941. 
SYNOPSIS TOC (Word Style "SN_Synopsis_TOC"). If you are submitting your paper to a journal that requires a synopsis graphic and/or synopsis paragraph, see the Instructions for Authors on the journal's homepage for a description of what needs to be provided and for the size requirements of the artwork.

Authors are required to submit a graphic entry for the Table of Contents (TOC) that, in conjunction with the manuscript title, should give the reader a representative idea of one of the following: A key structure, reaction, equation, concept, or theorem, etc., that is discussed in the manuscript. Consult the journal's Instructions for Authors for TOC graphic specifications.

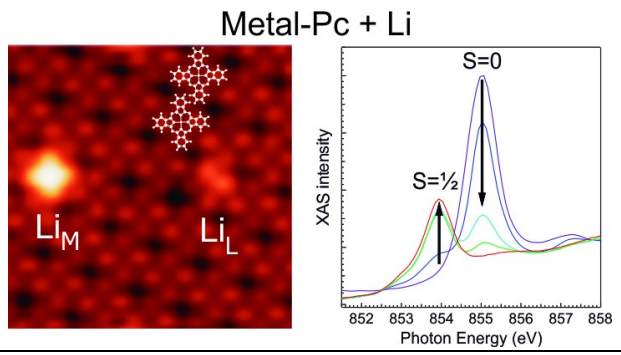

